\title{
Doping Approaches for Organic Semiconductors
}

\author{
Alberto D. Scaccabarozzi,${ }^{1{ }^{* *}}$ Aniruddha Basu, ${ }^{1 \dagger}$ Filip Aniés,${ }^{3}$ Jian Liu, ${ }^{2}$ Osnat Zapata-Arteaga, ${ }^{4}$ \\ Ross Warren, ${ }^{5}$ Yuliar Firdaus,,${ }^{1,7}$ Mohamad Insan Nugraha, ${ }^{1}$ Yuanbao Lin, ${ }^{1}$ Mariano Campoy- \\ Quiles ${ }^{4}$ Norbert Koch, ${ }^{5}$ Christian Müller, ${ }^{2}$ Leonidas Tsetseris, ${ }^{6}$ Martin Heeney, ${ }^{3}$ Thomas D. \\ Anthopoulos ${ }^{1 *}$
}

1. King Abdullah University of Science and Technology (KAUST), KAUST Solar Center (KSC), Thuwal 23955, Saudi Arabia

2. Department of Chemistry and Chemical Engineering, Chalmers University of Technology, Göteborg 412 96, Sweden

3. Department of Chemistry and Centre for Processable Electronics, Imperial College London, London, W12 0BZ, UK

4. Materials Science Institute of Barcelona, ICMAB-CSIC, Campus UAB, 08193 Bellaterra, Spain

5. Helmholtz-Zentrum Berlin für Materialien und Energie GmbH, Kekulé-Strasse 5, 12489 Berlin, Germany 
Institut für Physik \& IRIS Adlershof, Humboldt-Universität zu Berlin, 12489 Berlin, Germany

6. Department of Physics, National Technical University of Athens, Athens GR-15780, Greece

7. Research Center for Electronics and Telecommunication, Indonesian Institute of Science, Jalan Sangkuriang Komplek LIPI Building 20 level 4, Bandung 40135, Indonesia

$\dagger$ Authors contributed to this work equally

Corresponding authors: thomas.anthopoulos@kaust.edu.sa; alberto.scaccabarozzi@kaust.edu.sa 

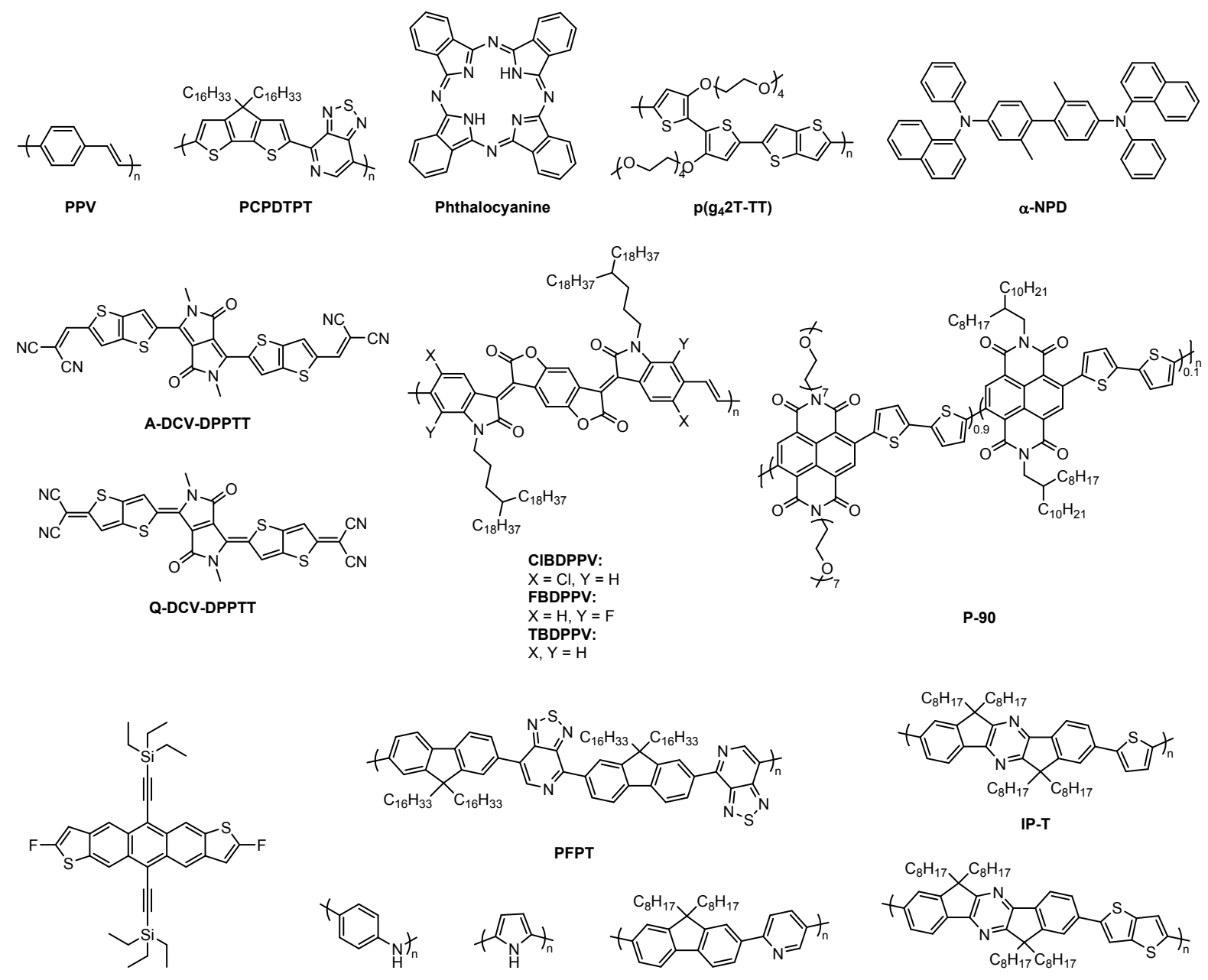

diF-TESADT

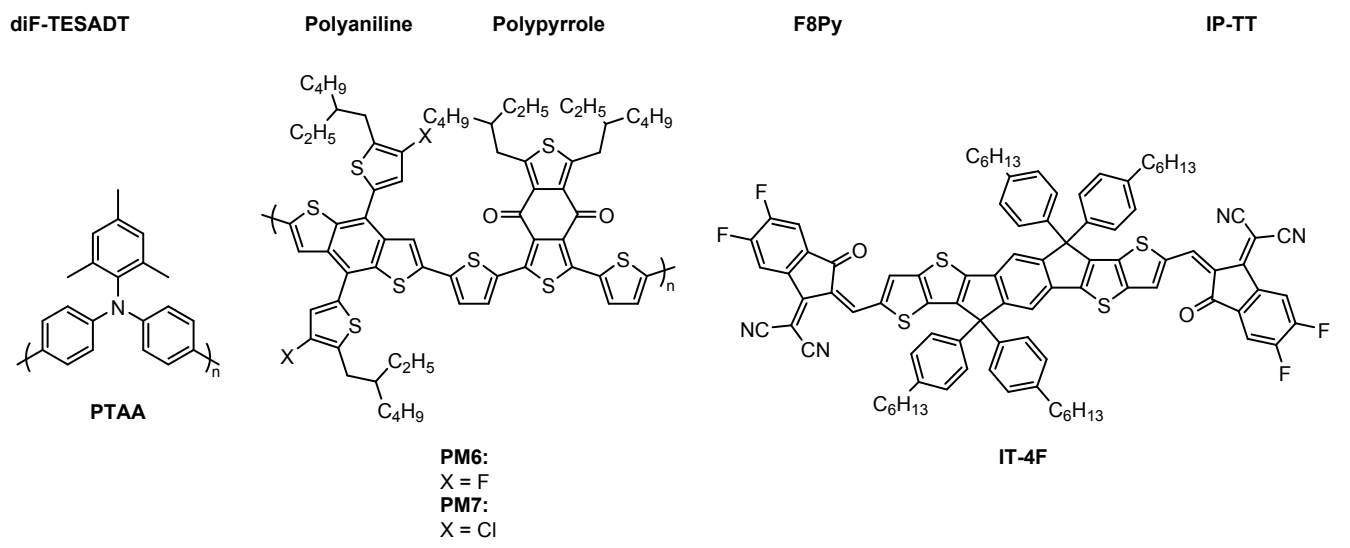

F8Py

IP-TT

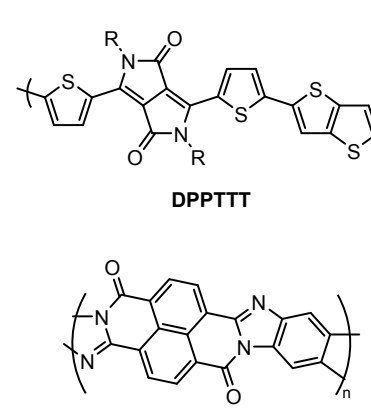

BBL

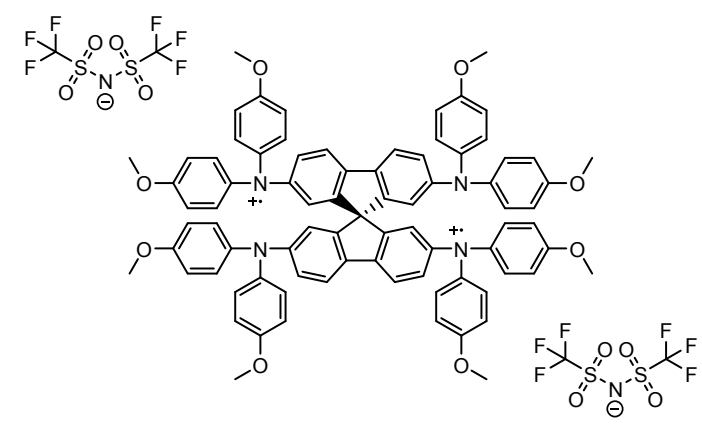

Spiro-OMe $\mathbf{S A b}+\underline{\mathbf{1}} \mathrm{sI})_{2}$

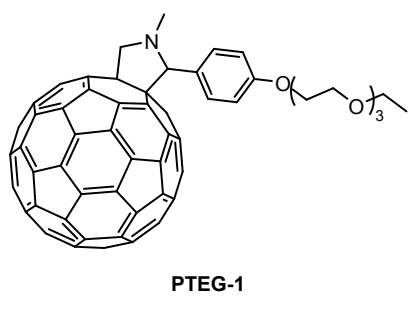

PTEG-1 

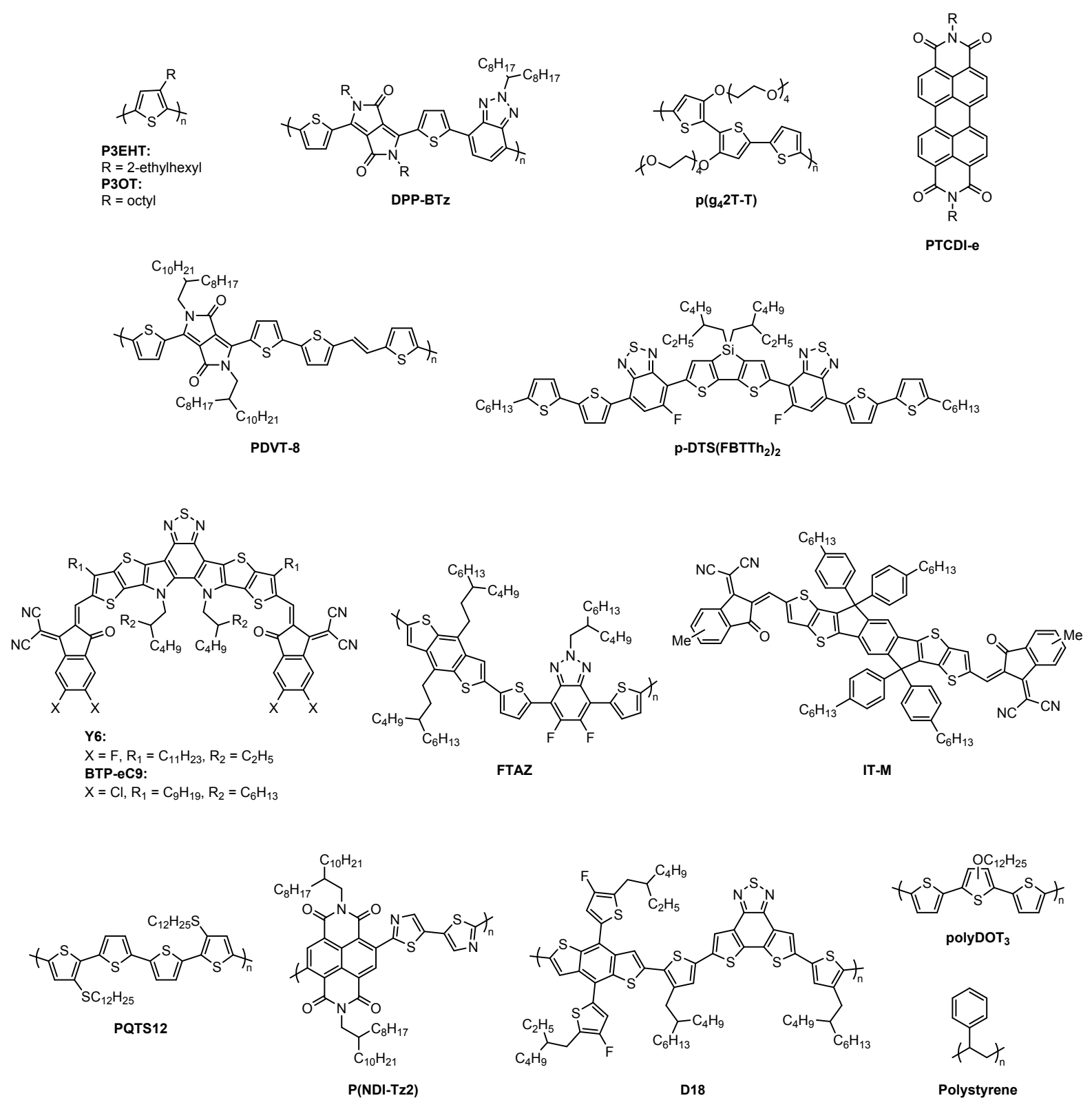

P(NDI-Tz2)

D18

Polystyrene
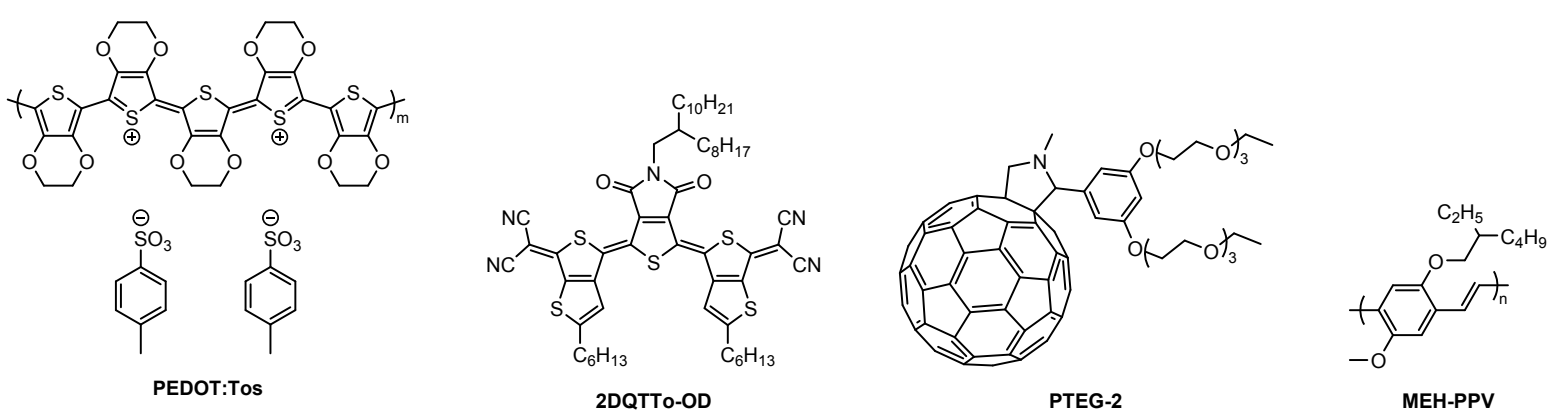

MEH-PPV 
Figure S1: Chemical structure of OSCs mentioned in the main text.

Dopants full names:

F-TCNQ: 2,3,5,6-tetra-fluoro-7,7,8,8-tetracyanoquinodimethane

Magic Blue: tris(4-bromophenyl)ammoniumyl hexachloroantimonate

EBSA: 4-ethylbenzenesulfonic acid

TAM: Triaminomethane

N-DMBI: (4-(1,3-dimethyl-2,3-dihydro-1H-benzoimidazol-2-yl)phenyl)dimethyl-amine

$\mathbf{B F}_{3}$ : boron trifluoride

F-TCNNQ: hexafluorotetracyanonaphthoquinodimethane

CN6-CP: hexacyano-trimethylene-cyclopropane

TBAF: tetrabutylammonium fluoride

BCF: tris(pentafluorophenyl)borane

ZnCF: bis(pentafluorophenyl)zinc

TTN: tetrathianaphthacene

BEDT-TTF: Bis(ethylenedithio)tetrathiafulvalene

BTQBT: bis(1,2,5-thiadiazolo)-p-quinobis(1,3-dithiole)

(RuCp*mes) $)_{2}$ : pentamethylcyclopentadienyl mesitylene ruthenium dimer

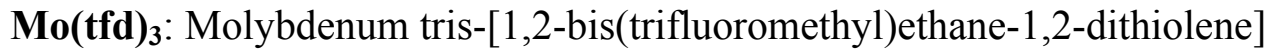

Mo(tfd-CO $\left.\mathbf{C O}_{2} \mathbf{M e}\right)_{3}$ : $\quad$ Molybdenum $\quad$ tris(1-(methoxycarbonyl)-2-(trifluoromethyl)ethane-1,2dithiolene)

Mo(tfd-COCF $\left.)_{3}\right)_{3}: \quad$ Molybdenum tris[1-(trifluoroethanoyl)-2-(trifluoromethyl) ethane-1,2dithiolene]

$\mathbf{C r}_{\mathbf{2}}$ (hpp) $)_{4}$ : tetrakis(1,3,4,6,7,8- hexahydro-2H-pyrimido[1,2-a]pyrimidinato)dichromium (II)

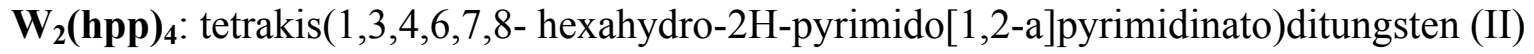

Ru(terpy) 2 : bis(2,2':6',2"-terpyridine)ruthenium

$\mathbf{R u}(\mathbf{t}-\text { but-terpy })_{2}$ : bis(4,4',4"-tri-tert-butyl-2,2':6',2"-terpyridine)ruthenium

CoCp 2 : bis(cyclopentadienyl)cobalt(II)

$\mathbf{R h}\left(\mathbf{C}_{5} \mathbf{H P h}_{4}\right)_{2}$ : bis(tetraphenylcyclopentadienyl)rhodium(II) 
$\mathbf{B F}_{\mathbf{3}}$ : Boron trifluoride

AlMe $_{3}$ : Trimethylaluminium

$\mathbf{B B r}_{3}$ : Boron tribromide

TBАОН: tetrabutyl ammonium hydroxide

TMCN3-CP: trimethyl 2,2',2”-(cyclopropane-1,2,3-triylidene)-tris(cyanoacetate)

PEI: polyethylenimine

BBL: poly(benzoimidazobenzophenanthroline)

TDAE: tetrakis(dimethylamino)ethylene

DMBI-BDZC:

(12a,18a)-5,6,12,12a,13,18,18a,19-octahydro-5,6-dimethyl-13,18[1',2']-benzenobisbenzimidazo[ $\left.1,2-b: 2^{\prime}, 1^{\prime}-d\right]$ benzo[i] $[2.5]$ benzodiazocine

DBU: amidines 1,8-diazabicyclo[5.4.0]undec-7-ene

DBN: diazabicyclo(5.3.0)non-5-ene

TBD: 1,5,7-triazabicyclo[4.4.0]dec-5-ene

DQ: diquat

BV: benzyl viologen

NHC: N-heterocyclic carbine

DMImC: 1,3-dimethylimidazolium-2-carboxylate

TPFB: tetrakis(pentafluorophenyl)borate

TrTPFB: trityl tetrakis(pentafluorophenyl) borate

DDB: Dodecaborane

PTPADT-SO ${ }_{3}$ Na: poly(4-(3'-sulfonatepropoxy-phenyl)bis(4-phenyl)amine-alt-2,2'-bithiophen) sodium

CPDT-BT: cyclopenta-[2,1-b;3,4-b']-dithiophene-alt-4,7-(2,1,3-benzothiadiazole)

FPI: fulleropyrrolidinium iodide

BDOPV: benzodifurandione-centered oligo(p-phenylene vinylene)

TmPyPB: 3,3' -(5' -(3-(pyridin-3-yl)phenyl)-[1,1' $: 3^{\prime} \quad, 1^{\prime \prime} \quad$-terphenyl]-3,3" -diyl)dipyridine

NOSbF $_{6}$ : Nitrosonium hexafluoroantimonate

EBSA: 4-ethylbenzenesulfonic acid

EBSAc: EBSA capped with an $o$-nitrobenzyl capping moiety 
DBSA: dodecylbenzenesulfonic acid

CSA: camphorsulfonic acid

DMDBS: 1,3:2,4-bis(3,4-dimethylbenzylidene)sorbitol

TFP: tetrafluorophthalonitrile

OFN: octafluoronaphthalene

TTF: Tetrathiafulvalene

TMTSF: tetramethyltetraselenafulvalene

DDQ: 2,3-dichloro-5,6-dicyano-1,4-benzoquinone

TMA: 3-(trimethoxysilyl)propan-1-amine

TEDA: $N$-(3-(triethoxysilyl)propyl)ethane-1,2-diamine)

CsF: Cesium fluoride

TBAPF $_{6}$ : tetrabutylammonium hexafluorophosphate

BSA: benzenesulfonic acid

TTF-TCNQ: tetrathiafulvalene-tetracyanoquinodimethane

N-DPBI: 4-(1,3-dimethyl-2,3-dihydro-1H-benzoimidazol-2-yl)-N,N-diphenylaniline

o-MeO-DMBI: 2-(2-Methoxyphenyl)-1,3-dimethyl-1H-benzoimidazol-3-ium 
Organic Semiconductors full names:

P3HT: poly(3-hexylthiophene)

4T: quaterthiophene

PBTTT: poly[2,5-bis(3-alkylthiophen-2-yl)thieno(3,2-b)thiophene]

PPV: poly(p-phenylene vinylene)

PCPDTPT: Poly[2,6-(4,4-bis-(2-ethylhexyl)-4H-cyclopenta $\quad\left[2,1-b ; 3,4-b^{\prime}\right]$ dithiophene)-alt4,7(2,1,3-benzothiadiazole)]

PCPDTBT: Poly[2,6-(4,4-bis-(2-ethylhexyl)-4H-cyclopenta $\quad$ [2,1-b;3,4-b']dithiophene)-alt4,7(2,1,3-benzothiadiazole)]

NDI: naphthalenediimide

$\mathbf{C}_{60}:$ Fullerene- $\mathrm{C}_{60}$

p(g42T-TT): poly(3,3'-bis(tetraethylene glycol methyl)-2,2'-dithiophene-thienothiophene)

p(g,2T-T): poly(3,3'-bis(tetraethylene glycol methyl)-2,2'-dithiophene-thiophene)

a-NPD: 2,2'-Dimethyl- $N, N^{\prime}$-di-[(1-naphthyl)- $N, N^{\prime}$-diphenyl]-1,1'-biphenyl-4,4'-diamine

PDPP(6-DO) 2 TT: poly[3,6-(dithiophene-2-yl)-2,5-di(6-dodecyloctadecyl)pyrrolo[3,4-c]pyrrole1,4-dione-alt-thieno[3,2-b]thiophene]

PCBM: [6,6]-Phenyl $\mathrm{C}_{61}$ butyric acid methyl ester

diF-TESADT: 2,8-Difluoro-5,11-bis(triethylsilylethynyl)anthradithiophene

TIPS-pentacene: 6,13-Bis(triisopropylsilylethynyl)pentacene

C16 $_{16}$ IDT-BT: indacenodithiophene-co-benzothiadiazole with copo- lymer with hexadecyl alkyl chains

PFPT: poly-fluorene-pyridylthiadiazole

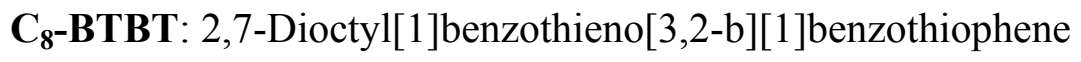

P(NDI2OD-T2): $\quad$ Poly $\left\{\left[\mathrm{N}, \mathrm{N}^{\prime}\right.\right.$-bis(2-octyldodecyl)-naphthalene-1,4,5,8-bis(dicarboximide)-2,6diyl]-alt-5,5'-(2,2'-bithiophene) $\}$

DCV-DPPTT: dicyanovinyl- dipyrrolo[3,4-c]pyrrole-1,4-iylidene)bis(thieno[3,2b]thiophene 
CIBDPPV: Poly[[5-chloro-1,2-dihydro-1-(4-octadecyldocosyl)-2-oxo-3H-indol-6-yl-3-ylidene](1E)-1,2-ethenediyl[5-chloro-1,2-dihydro-1-(4-octadecyldocosyl)-2-oxo-3H-indol-6-yl-3ylidene][2,6-dioxobenzo[1,2-b:4,5-b']difuran-3,7(2H,6H)-diylidene]]

F8Py: Poly[(9,9-dioctylfluorenyl-2,7-diyl)-alt-(2,6-pyridine)]

PTAA: Poly[bis(4-phenyl)(2,4,6-trimethylphenyl)amine], Poly(triaryl amine)

PBDB-TF: $\quad$ Poly[(2,6-(4,8-bis(5-(2-ethylhexyl-3-fluoro)thiophen-2-yl)-benzo[1,2-b:4,5b']dithiophene))-alt-(5,5-(1',3'-di-2-thienyl-5',7'-bis(2-ethylhexyl)benzo[1',2'-c:4',5'c']dithiophene-4,8-dione)]

IT-4F: 3,9-bis(2-methylene-((3-(1,1-dicyanomethylene)-6,7-difluoro)-indanone))-5,5,11,11tetrakis(4-hexylphenyl)-dithieno[2,3-d:2',3'-d']-s-indaceno[1,2-b:5,6-b']dithiophene

BBL: Poly(benzimidazobenzophenanthroline)

PTEG-1: triethylene glycol monoethyl ether fulleropyrrolidine

P3EHT: poly(3-(2'-ethyl)hexylthiophene)

DPP-BTz: poly[[2,5-bis(2-octadecyl)-2,3,5,6-tetrahydro-3,6-diketopyrrolo[3,4-c]pyrrole-1,4diyl]-alt-(2-octylnonyl)-2,1,3-benzotriazole]

P3OT: Poly(3-octylthiophene-2,5-diyl)

PEDOT:PSS: poly(3,4-ethylenedioxythiophene) polystyrene sulfonate

DPPTTT: Poly[2,5-(2-octyldodecyl)-3,6-diketopyrrolopyrrole-alt-5,5-(2,5-di(thien-2-yl)thieno [3,2-b]thiophene)]

p-DTS(FBTTh $)_{2}$ : 7,7'-[4,4-Bis(2-ethylhexyl)-4H-silolo[3,2-b:4,5- $\left.b^{\prime}\right]$ dithiophene-2,6-diyl]bis[6fluoro-4-(5'-hexyl-[2,2'-bithiophen]-5-yl)benzo[c][1,2,5]thiadiazole $]$

FBDPPV: Poly[[7-fluoro-1,2-dihydro-1-(4-octadecyldocosyl)-2-oxo-3H-indol-6-yl-3-ylidene](1E)-1,2-ethenediyl[7-fluoro-1,2-dihydro-1-(4-octadecyldocosyl)-2-oxo-3H-indol-6-yl-3ylidene](2,6-dioxobenzo[1,2-b:4,5-b']difuran-3,7(2H,6H)-diylidene)]

PQTS12: poly(bisdodecylthioquaterthiophene)

PTCDI-e: N,N'-di( (Z)-9-octadecene)-3,4,9,10-perylene tetracarboxylic diimide

Y6: 2,2'-((2Z,2'Z)-((12,13-bis(2-ethylhexyl)-3,9-diundecyl-12,13-dihydro-[1,2,5]thiadiazolo[3,4e]thieno[2",3':4',5']thieno[2',3':4,5]pyrrolo[3,2-g]thieno[2',3':4,5]thieno[3,2-b]indole-2,10diyl)bis(methanylylidene))bis(5,6-difluoro-3-oxo-2,3-dihydro-1H-indene-2,1diylidene))dimalononitrile 
IT-M: 3,9-bis(2-methylene-((3-(1,1-dicyanomethylene)-6/7-methyl)-indanone))-5,5,11,11tetrakis(4-hexylphenyl)-dithieno[2,3-d:2',3'-d']-s-indaceno[1,2-b:5,6-b']dithiophene

P(NDI-Tz2): Poly $\left\{\left[\mathrm{N}, \mathrm{N}^{\prime}\right.\right.$-bis(2-octyldodecyl)-naphthalene-1,4,5,8-bis(dicarboximide)-2,6-diyl]alt-5,5'-(2,2'-bithiazole $)\}$

BTP-eC9: 2,2'- [[12,13-Bis(2-butyloctyl)-12,13-dihydro-3,9dinonylbisthieno[2", 3":4',5'] thieno[2',3':4,5]pyrrolo[3,2-e:2',3'-g][2,1,3]benzothiadiazole-2,10diyl]bis[methylidyne(5,6-chloro-3-oxo-1H-indene-2,1(3H)-diylidene) ]]bis[propanedinitrile] $\operatorname{polyDOT}_{3}: \operatorname{poly}\left(\beta^{\prime}-\right.$ dodecyloxy- $\alpha, \alpha^{\prime},-\alpha^{\prime}, \alpha^{\prime \prime}$ terthienyl $)$

MEH-PPV: Poly[2-methoxy-5-(2-ethylhexyloxy)-1,4-phenylenevinylene] BTP: dithieno[2 $\left.2^{\prime}, 3^{\prime}{ }^{\prime}: 4^{\prime}, 5^{\prime}\right]$ thieno[2 $\left.2^{\prime}, 3^{\prime}: 4,5\right]$ pyrrolo[3,2-e:2 $2^{\prime}, 3^{\prime}$ $g][2,1,3]$ benzothiadiazole

BO: 2-bultyloctyl 